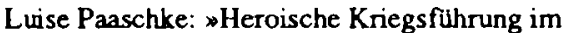
männlichen Kulturalter*, In: Die Deutsche Kämpferin, Februar 1935, S. 324-326.

28 Sophie Rogge-Bömer: Liberalismus. In: Die deutsche Kämpferin, September 1933, S. 97.

29 Ebd. S. 100.

30 Sophie Philipps: Dr. Hans Beyer - Die Frau in der politischen Entscheidung. In: Die deutsche Kämpferin, April 1934, S. 30.

31 Vgl. etwa Annemarie Doherr: Deutschland und Europa - Genfer Ergebnisse. In: Die deutsche Kämpferin, November 1934, S. 241-243.

32 Sophie Rogge-Börner: Brief an die Chefredaktion der Zeitung Politiken $*$. In: Die deutsche Kämpferin, November 1934, S. 247-248.

33 Sophie Rogge-Bömer: Liberalismus..., a.a.O., S. 98.

34 Sophie Rogge-Bömer: Die Ura-Linda-Chronik. In: Die deutsche Kämpferin, April 1934. S. 7.

35 In der vorletzten Nummer von Die deutsche Kampferin, April 1937, veröffentlicht Sophie Rogge-Börner weite Teile des Werks von Heinz Brdcher: Ernst Haeckels Bluts- und Geisteserbe, Munchen 1936.

Andrea Wicke

\section{Marquis de Sade: Justine oder Die Leiden der Tugend, gefolgt von Juliette oder Die Wonnen des Lasters (1797)}

\section{Anmerkungen zu einem nicht mehr ganz neuen Thema}

In ihrem Buch Pornographie - Männer beherrschen Frauen hat Andrea Dworkin den Stab über den französischen Marquis aus dem 18. Jahrhundert gebrochen. Sie schreibt:

Er war Vergewaltiger und Autor in einer Person - beide Stränge seines Wesens sind verflochten zu einem Gewebe aus Scheußlichkeit. Sein Leben und Schreiben waren aus einem Stück, ein ganzes Tuch getränkt mit dem Blut erdachter und realer Frauen. ${ }^{2}$

Dworkins Metaphorik ist gnadenlos: Bilden Leben und Text erst einmal ein Stück Stoff, und ist dieses vollgesogen mit dem Blut der weiblichen Opfer, dann gerăt die Lektüre mindestens zur Befleckung, wenn nicht zur Besudelung (der feministischen Unschuld?). Damit wird jedes Interesse, selbst das kleinste Fünkchen Leselust als illegitime Form der Neugierde diskreditiert. Donatien Alphonse Francois Marquis de Sade wird seine neuerliche Verdammung wohl kaum mehr stơren - aktuell angeklagt sind denn auch die Leserinnen: Mit welchem Recht interessieren sie die Phantasien dieses Autors und nicht vielmehr nicht?? - In der stickigen Atmosphăre des totalen Rechtfertigungszwanges entlastete Silvia Bovenschen die Angeklagten mit der Feststellung: »Auf falsche Fragen gibt es keine richtigen Antworten." Die Argumentation Dworkins und der an diese anknüpfenden Anti-Pomo-Kampagne resümierend, kommt sie zum ernüchterten und skeptischen Fazit:

Es bleibt anzumerken, daß fast alles, was jetzt publizistisch aufgewirbelt wird, in anderen Konstellationen schon mal gesagt wurde. So reißerisch sie in den Medien aufgemacht sein mag, es kann nichts darüber hinwegtäuschen - diese Diskussion ist im Grunde langweilig. Liegt das daran. daß sich unser Denken und Sprechen über Sexualität zu sehr in eingefahrenen Bahnen bewegt? Müßten neue Formen und Wege gefunden werden, über sie zu sprechen und zu denken? Und möglicherweise auch, mil ihr umzugehen. ${ }^{3}$

Neue Formen und Wege... - gern. Aber wie? - Zunăchst ist der Ratschlag, den Bovenschen uns im Namen Michel Foucaults mit auf den Weg gibt, zu beachten: Hören wir auf, den »Sex als das allumfassende 
Geheimnis« zu entziffern. Aus dieser Perspektive erubrigt sich dann eventuell auch der Druck zur eindeutigen Diagnose, die scheinbar eine jede Lektüre pornographischer Phantasie beenden können muß. Dies Phănomen wirkt m.E. auch dort, wo der Marquis de Sade als Sadist, Sexualpathologe, Enzyklopådist, Soziologe und Aufklärer gelesen wird.

Aber fehlen nicht Lesarten, die der Eigengesetzlichkeit assthetischer und rhetorischer Verfahren in seinen Romanen ihr Recht geben? Anders - aus rezeptionsästhetischer Perspektive - gefragt: Welche Erfahrungen können Leserinnen heute machen mit Justine und Juliette und den Bildern, die sie von sich zu haben gewohnt sind? Ist es z.B. nicht vielversprechender und vielleicht auch vergnüglicher - der "wollüstigen Sehnsucht nach Auslosschung des eigenen Bewußtseins ${ }^{4}$ einmal in anderer Form zu folgen, als in der Analyse der sexuellen Praktiken und ihrer theoretischen Legitimation? Simone de Beauvoir, Angela Carter, Monika Treut und zuletzt meines Wissens Elisabeth Lenk - um nur wenige zu nennen - haben interessante und kluge Arbeiten zu de Sades Texten geschrieben und sich meist auch zur Rolle des Weiblichen darin geăußert. Im folgenden sollen einmal eher nicht reprăsentative und oft verstreute Momente dieser Arbeiten Beachtung finden. Gemeinsam ist ihnen, daß sie aufmerksam machen auf ästhetische Qualităten des Romans und eine Eigendynamik seiner Frauengestalten, die nicht aufgeht in ihrer găngigen Attribuierung. An den Aporien einer identitătslogischen Interpretation entschärfen sich hăufig präzise Einzelbeobachtungen. Insbesondere Justine und Juliette werden als polarisierte weibliche Geschlechtscharaktere rezipiert: Tugendsam die eine, gottlos die andere Frau. ${ }^{5}$

Ein zweiter Topos der de Sade-Forschung besteht in der Rede von der Unlesbarkeit seiner Werke. ${ }^{6}$ Gelesen wird deshalb meist nur unter dem Etikett Pflichtlektüre. Dann - ich formuliere überspitzt - subsumiert sich witterndes Schmö- kern unter die mehr oder weniger treffsichere Jagd auf Belegstellen. Dagegen hat jüngst Elisabeth Lenk protestiert mit dem berechtigten Hinweis auf die "dialogische Struktur « ${ }^{7}$ des Romans.

Anläßlich der falschen Fragen und Anklagen, die Andrea Dworkin dem de Sadeschen Werk und seinen Figuren zumutet, scheint es denn auch nicht unnötig, eine vergleichsweise banale Feststellung zu treffen: Ein literarischer Text ist nicht gleich der Summe seiner Prädikate. Weder die Justine- noch die Juliette-Figur werden angemessen verstanden, wenn sie auf ihre dramaturgische Funktion in einer Reihe sexueller Szenerien reduziert werden. Ebensowenig gehen sie auf in der Attitude ihres Geschlechtercharakters. Interessantere Aufschlüsse über die schillernden Rollen des Weiblichen verspricht eine nichtidentifikatorische Analyse des Romans. Deshalb meine Frage: Könnte in dem Verdikt unlesbar die Erinnerung an eine irritierende Erfahrung enthalten sein, wie sie schon Simone de Beauvoir anläßlich ihrer Sade-Lektüre festhielt? Ich zitiere:

Seine Gedankengänge sind darauf angelegt, den in die Irre zu führen, der sie festlegen will, und damit erreicht er, was er will: uns beunruhigen. Sogar seine formalen Ausdrucksmittel sind dazu angetan. uns zu verwirren. Er spricht mit monotoner gehemmter stimme, beginnt uns zu langweilen, aber plötzlich blitzt bitter. spöttisch, obszön eine Wahrheit auf, die diese Eintönigkeit erhellt und durch sie noch schärfer akzentuiert wird. ${ }^{8}$

Simone de Beauvoir vemeinte ihre schon 1955 gestellte Frage "Soll man de Sade verbrennen? $\ll$. Ihre damaligen Gedankengänge zu seinem Leben und seinen Schriften sind von dem Bemühen geleitet, den Autor vor vorschnellen Vereindeutigungen zu schützen. Am Beginn ihres Essays steht eine Beobachtung, die für die Vorsichtigkeit ihrer Annäherungen bestimmend 
bleibt: »Uns fesseln seine Bücher, sobald wir erkennen, daß er mittels seiner ermüdenden Wiederholungen, klischeehaften Formulierungen und stilistischen Ungeschicklichkeiten eine Erfahrung mitzuteilen versucht, deren Eigenart es gerade ist, nicht mitteilbar zu sein. ${ }^{9}$ Es ist bisher vernachlässigt worden, diesen Überschuß an Unartikulierbarkeit in Beziehung zu den Romanfiguren zu setzen. War de Sade mit Hilfe weiblich konnotierter Hauptrollen etwas zu artikulieren möglich, was er mit einem mănnlichen Helden nicht hătte mitteilen können? Welche erzählerischen Freiheiten entstehen durch die zentrale Stellung eines weiblichen Geschwisterpaares? Außer Justine und Juliette interessieren auch die listige Clairwil, das ist Juliettes langjăhrige Freundin und die merkwürdige Erscheinung der Zauberin Durand. De Beauvoir notierte zur letzteren: "Die Außergewơhnlichste aller seiner Frauengestalten, die Durand, versieht er in seiner Phantasie mit einem riesigen Kitzler, der es ihr ermöglicht, sich geschlechtlich wie ein Mann zu verhalten. ${ }^{10}$ Man darf - denke ich - diese Ausdrucksmöglichkeiten der Figur weder unterschătzen, noch ihr abweichendes Verhalten sogleich ins »Androgyn-Harmlose « ${ }^{11}$ auflösen. Ware es demgegenüber nicht sinnvoller, den ästhetischen Spielraum des Weiblichen offenzuhalten? Genauer ist zu prüfen, was die permanenten anatomischen Verzerrungen der Menschen im Roman von 1797 meinen können, wathrend sich doch zur gleichen Zeit die anthropologischen Konzeptionen des Menschen - und in nachhaltigster Weise der Frau - auf seine biologische Geschlechtlichkeit zu fixieren begannen: "Anatomie als Schicksal «" hieß das Motto dort.

An dieser Stelle ist das 1984 erschienene Buch "Die grausame Frau« von Monika Treut zu erwăhnen. Treut untersucht die literarischen Darstellungen grausamer Frauen bei de Sade und Leopold von Sacher-Masoch. Hier kommt lediglich der erste Teil ihrer Ausführungen in Betracht. Ich zitiere aus dem Vorwort:
Vielleicht bietet eine Dekonstruktion dieser Bilder (grausamer Frauen, A.W.) nicht nur ein besseres Wissen, sondern auch eine Möglichkeit, die den Frauen ein neues Feld eröffnen könnte? (...) Ein souveränes Spiel, das Lust bereitet, weil es gegen jede feste Rolle eine, Grausamkeite stellt, die nur als Vexierbild gelesen und gelebt werden dann. ${ }^{13}$

Anspruchsvolle Perspektiven also sind es, die hier entworfen werden. Aber die Verfasserin hält ihrem eigenen Anspruch nicht stand. ${ }^{14}$ Zunăchst sei ein methodischer Mangel benannt: Der im Vorwort angemeldete Anspruch auf Dekonstruktion hătte eine minutiơse Lektüre des vertrackten Doppelromanes erfordert. Denn der Terminus meint - im Sinne Paul de Mans ${ }^{15}$ primăr einen Vorgang, der im Text und seinen rhetorischen Bedeutungsstrukturen angelegt ist. Die spannenden Moglichkeiten dieses Ansatzes negiert Treut, wenn sie von Juliette oder die Wonnen des Lasters behauptet, es handele sich dabei gar nicht um eine "zielgerichtete Romanform " (?, A.W. ${ }^{16}$. (Justine oder Die Leiden der Tugend interessieren Treut nicht.) Ihre zentrale These geht aus vom Sadeschen Programm eines Tout dire. Unter verschiedenen Aspekten werden die zahlreichen Tabuverletzungen der Handlung thematisiert (»Gegen den guten Geschmack«, »Gegen die normale Sexualită «, »Gegen die mănnliche Anatomie«...), doch bezüglich der grausamen Protagonistin zieht die Autorin ein eher deprimierendes Fazit: "Juliette wird grausam, gerade weil sie eine Frau ist. " ${ }^{17}$ Ihr sozialer Status als weiblicher Libertin sei der einer Freigelassenen in einer weiterhin von männlichen gleich grausamen Konventionen bestimmten Lebenswelt. $\mathrm{Zu}$ überleben ist hier nur in Form von Anpassung. Mit den Worten Monika Treuts: "Hinter der Maske der grausamen Frau de Sades sehen wir die unterdrückte Frau, deren wirkliche Souverănităt noch immer Utopie ist. ${ }^{18}$ Da bleibt kein Ort für ein souveränes Spiel, das Leserinnen Vergnügen bereiten könnte. Die 
inhaltlichen Konsequenzen des eingangs genannten Paradigmas des Vexierbildes werden von Monika Treut nicht durchgeführt. ${ }^{19}$ Juliette wird eben doch eine feste Rolle zugewiesen: Sie ist die aufgrund ihrer verwerflichen Mimikry an mănnliche Standards arrivierte Frau.

Erwăhnen mơchte ich noch Angela Carters groBeren Essay »The Sadeian Woman «, der 1981 unter dem Titel "Sexualităt ist Macht " auf deutsch erschien. Die Stărken dieser Autorin liegen in der geistreich paraphrasierenden Darstellung der zentralen Handlungsstrănge von Justine und Juliette. Carter bietet dabei eine Fülle unsystematischer Assoziationen, die anregend $\mathrm{zu}$ lesen sind. Ihnen fehlt der ideologische Gestus, der so oft die Forschung zu de Sade begleitet. Auf diese Weise entstehen Reibungspunkte, an denen man nachhaken und nachprüfen möchte, die also neugierig machen auf den Autor und sein Werk. Zum Beispiel folgender Auszug:

Sades sexuelle Metaphern sind stets zweideutig. Durch seinen Sprachgebrauch mystifiziert er die sexuellen Merkmale des weiblichen Körpers. Er beschreibt ihn mit sakralen Wendungen, ja mit Begriffen der sakralen Architektur, als ob er ein heiliger Ort sei. (...) Diese ironische Sakralisierung durchzieht das ganze Werk Sades. Noch in der tiefsten Demütigung, wenn er mit Blut und Kot besudelt ist, behält der Altar seine perfide Magie. ${ }^{20}$

Außer den zweideutigen metaphorischen Formulierungen erwahnt Carter auch das »komplexe dialektische Verhăltnis « ${ }^{21}$ von Justine und Juliette. Bei ihr finden sich sogar einige Hinweise zur Gattungsproblematik dieses Textes. Sie bezeichnet mehrmals die Geschichte der Juliette als "pikareske Erzăhlung « oder »Schelmenroman “. ${ }^{2}$ Dieser Gedanke wird leider weder hergeleitet, noch weiter verfolgt, geschweige denn für die Gestalt der Juliette fruchtbar gemacht. Er könnte aber dazu beitragen, die Herkunft ihrer reichhaltigen
Ausdrucks- und Verhaltensmogglichkeiten zu klären.

Elisabeth Lenk, mit deren Aufsatz ich diesen notwendigerweise fragmentarischen Literaturbericht dann auch beschlieBen mochte, führt ebenfalls gattungsspezifische Gründe für die Außergewöhnlichkeit der de Sadeschen Frauengestalten an. Unter den Titel »JUSTINE und JULIETTE - Antagonistisches Frauenbild und antagonistische Moral bei D.A.F. de Sade« plädiert sie dafür, de Sade als Soziologen zu lesen, genauer: als "Pathologen der Macht « ${ }^{23}$. Überzeugend leitet die Autorin den schockierenden Effekt seiner Schriften $a b$ von der Perspektive eines Mannes, der vom »jubelnden Männerbund ${ }^{24}$ der Aufklärung ausgeschlossen war. Lenk setzt den Akzent auf die »strukturelle Gewalt ${ }^{25}$, die in den Prosaschriften zur Sprache komme. Zur Konzeption von $J u$ stine (...) gefolgt von Juliette (...) schreibt sie:

Schon von seiner Konstruktion her ist unser Doppelroman ein Meisterstück des Sadismus. Nicht genug, das Justine von Unglück zu Unglück geführt wird; am Ende ihres jungen Lebens muß sie sich auch noch den Roman Juliettes, ihrer ungleichen Schwester, anhören, dies einzige Loblied auf das Laster. ${ }^{26}$

An anderer Stelle wird nicht nur - wie schon zu Anfang erwähnt - die dialogische Struktur des Romanes betont, sondern darüberhinaus anschaulich vorgeführt, da $\mathrm{B}$ eine stärkere Berïcksichtigung der Erzählstrategie zur Revision falscher Fronten in der Sekundärliteratur führen kann. Mit ihrer These schließlich, de Sade stelle im Schicksal des Geschwisterpaares »seine eigene Doppelheit ${ }^{27}$ dar, greift die Verfasserin eine Vermutung auf, die schon Simone de Beauvoir ${ }^{28}$ ăußerte: Gemeint ist die Beobachtung einer starken und äußerst ambivalent sich äußernden Affinität des Autors zu seinen Imaginationen des Weiblichen. Elisabeth Lenk akzentuiert dieses Moment hinsichtlich der Figuren auf entscheidene Weise neu. Anschließend an die 
exemplarische Kommentierung des einzigen Traumes im Doppelroman (Juliette trăumt von Justine) umschreibt sie sehr einprăgsam die - auch erzathltheoretisch eminente - Beziehung der beiden Gestalten zueinander: "Die Umrisse der beiden Frauengestalten verschwimmen. Sie gehen auf einmal ineinander über und bilden nur mehr ein einziges, moralisch-androgynes Doppelwesen, dessen eine Hälfte die andere trăumt. ${ }^{29}$ Korrespondenzen zwischen der Justine-Hălfte und dem Anteil der Juliette unterlaufen den Typus, den jede einzelne von ihnen repräsentieren soll. Die strukturelle Doppelung des Textes und seiner Figuren erlaubt es möglicherweise vom Weiblichen als Verfahren zu sprechen. Hier ist eine phantasievolle und skrupulose Lektüre gefordert, die den historischen und gattungstheoretischen $\mathrm{Be}$ dingungen der ästhetischen Ausdrucksmoglichkeiten nachgeht. Auch in dieser Hinsicht enthălt Elisabeth Lenks Essay wegweisende Implikationen. Ich spreche von einem Passus, in dem Justine und Juliette im Sinne rhetorischer Figuren verstanden werden. Sie können als Allegorien gelesen werden. Nicht unterschlagen werden soll dabei, daß Lenk die potentiell subversive Mehrdeutigkeit des Allegorischen sogleich wieder auf den eher affirmativen Aspekt der Personifikation reduziert. Die Stelle im Zusammenhang: »In der Art, wie de Sade diese beiden Gestalten behandelt, werden sie zu Allegorien, die eine der Tugend, die andere des Lasters. Justine ist der Inbegriff aller weiblichen Tugenden. ${ }^{30}$ Justine mag vom Marquis aus dem 18. Jahrhundert so intendiert gewesen sein, nur. Gelungen ist ihm das nicht. Ich plădiere dafür, die Spannung des Nicht-Identischen in den Konzeptionen des Weiblichen auszuhalten, um sie verheißungsvoll werden zu lassen.
Anmerkungen

1 Der Doppelroman ist zuletzt 1987 im GrenoVerlag, Nördlingen erschienen. Die Ausgabe enthalt einen Artikel zur Rezeption in Deutschland und eine ausfuhrliche Bibliographie von Michael Farin und Hans-Ulrich Seifert.

2 Andrea Dworkin: Pornographie - Männer beherrschen Frawen. Köln 1987, S. 88.

3 Silvia Bovenschen: Auf falsche Fragen gibt es keine richtigen Antworten. Anmerkungen zur Pornographie-Kampagne. In: Autonome Frauen. Schlüsseltexte der Neuen Frauenbewegung seit 1968. Hrsg. von Ann Anders. Ffm 1988, S. 266-279.

4 Susan Sontag: Die pornographische Phantasie. In: dies., Kunst und Antikunst. München/Wien 1980, S.58.

5 Dieser Strang reicht vom Kindler Literatur Lexikon uber Dworkin bis hin zu einer Elisabeth Lenk Andrea Dworkin: Pornographie - Männer beherrschen Frauen. A.a. O., S. 117. Elisabeth Lenk: JUSTINE und JULIETTE. Antagonistisches Frauenbild und antagonistische Moral bei D.A.F. de Sade. In: Streitbare Philosophie. Marghertia von Brentano zum 65. Geburtstag. Hrsg. von Gabriele Althaus, Irmingard Staeuble. Berlin 1988, S. 294, 296.

6 Schon Roland Barthes konstatiert dies Phänomen miBbilligend. Ich empfehle sein - oft geschmähtes Buch - in diesem Zusammenhang zur kritischen Kenntnisnahme. Roland Barthes: Sade, Fourier, Loyola Ffm. 1986, S. 43.

7 Elisabeth Lenk: A.a.O., S. 292.

8 Simone de Beauvoir: Soll man de Sade verbrennen? Munchen 1964, S. 50.

9 A.a.O., S. 10.

10 A.a.O., S. 36.

11 Diese Formulierung stammt von Cora Stephan: Heim zu Mama. Ein polemisches Nachwort zu Alice Schwarzers Anti-Porno-Kampagne. In: Die Zeit, Nr. 16-15.April 1988, S.70. Auch die hier besprochenen Arbeiten sind von diesem nivellierenden $Z$ ug nicht frei.

12 Weiterfuhrende Literatur zu diesem Thema bieten Claudia Honegger und Hans-Ulrich Seifert. Insbesondere: Claudia Honegger: Die Französische Anthropologie der Revolutionszeit und die Neubestimmung der Geschlechter. In: Sklavin oder Bürgerin? Französische RevoIution und Neue Weiblichkeit 1760-1830. Hrsg. von Viktoria Schmidt-Linsenhoff. Marburg 1989, S. 294-307. Die zitierte Formulienung ist zu finden in: Claudia Honegger. Überlegungen zu Michel Foucalts Entwurf einer Geschichte der Sexualität. Darmstadt (cinca) 1981, S. 14. 
Grundlegend fit das historische Umfeld des Autors ist: Hans-Ulrich Seifert: Sade, Leser und Autor. Ffm, Bern 1983. Zu diesem Aspekt siehe S. $331 \mathrm{f}$.

13 Monika Treut: Die grausame Frau. Basel/Ffm 1984, S. 8.

14 Die lakonische Selbsteinschätzung der Autorin stimmt mit meinem Urteil vermutlich liberein. Ihre Doktorarbeit aus dem Jahr 1982 nennt sie einen "Gewaltria durch die Welt der Wissenschaft« mit der sie eigentlich nur einen Studienabschluß habe machen wollen... Monika Treut: Die flambierte Küche. In: Pornost. Triebkultur und Gewinn. Hrsg. von Brigitte Classen. München 1988. S. 155. (Ich möchte darauf hinweisen, daB in diesem Sammelband u.a die hier erwähnten Aufsäıze von Silvia Bovenschen und Elisabeth Lenk wieder publiziert wurden.)

15 Zur Orientierung: Paul de Man: Allegorien des Lesens, Ffm. 1988.

16 Monika Treut: A.a.O., S. 39.
17 A.a.O., S. 97

18 A.a.O., S. 98

$19 \mathrm{Zu}$ literarischen Darstellungen des Weiblichen im Sinne von »Vexierbildern « schon Silvia Bovenschen im Zusammenhang mit Wedekinds Lulu: Silvia Bovenschen: Die imaginierte Weiblichkeit. Exemplarische Untersuchungen zu kulturgeschichtlichen und literarischen Präsentationsformen des Weiblichen. Ffm 1979, S. 58.

20 Angela Carter. Sexualität ist Macht. Die Frau bei de Sade. Reinbek 1981, S. 93.

21 A.a.O., S. 131.

22 A.aO., S. 102, 117, 144.

23 Elisabeth Lenk: A.a.O., S. $288 f$.

24 A.a.O., S. 287.

25 A.a.O., S. 289.

26 A.a.O., S. 295.

27 A.a. O., S. 296.

28 Simone de Beauvoir. A.a.O., S. 35f.

29 Elisabeth Lenk: A.a. O., S. 300.

30 A.a.O., S. 294. 